\title{
Study of propagation characteristics of outdoor-to-indoor channel in the 60-GHz band
}

\author{
CHENG Long ${ }^{1}$, BAO Wencheng ${ }^{1}$, LIU Ning ${ }^{1}$, YUE Guangrong ${ }^{1}$, ZOU Xianbing ${ }^{1}$, QIU Robert Caiming ${ }^{2}$
}

1. University of Electronics Science and Technology of China, Chengdu 611731, China

2. Tennessee Technological University, Cookeville TN 38505, USA

\begin{abstract}
The millimeter-wave frequency band $(30 \sim 300 \mathrm{GHz})$ has received significant attention. Millimeter-wave frequencies have been used for backhaul, cell communication, and other high speed communication. With the advent of $5 \mathrm{G}$ communication, millimeter-wave frequencies such as $60-\mathrm{GHz}$ band have been attracting attention as possible candidate for next-generation wireless networks. The traditional application for $60-\mathrm{GHz}$ band is point-to-point communication. Some typical scenarios have been cited in a recent $5 \mathrm{G}$ white paper. There exist some traditional channel models for $3 \mathrm{G}$ and $4 \mathrm{G}$ communication. However, 5G has a new channel model (the outdoor-to indoor channel model, or O2I) that has not been clearly studied. Some conventional channel measurements have been conducted for this new band. Two measurement systems in the 60-GHz band for penetration loss and small-scale measurement for different scenarios are presented. By analyzing our measurement data, we can prove that the O2I channel does not generate new paths and only add some material penetration loss.
\end{abstract}

Key words: $60 \mathrm{GHz}$ band, $5 \mathrm{G}$, millimeter wave, $\mathrm{O} 2 \mathrm{I}$, penetration loss, $\mathrm{O} 2 \mathrm{O}, \mathrm{InH}$.

Citation: CHENG L, BAO W C, LIU N, et al. Study of propagation characteristics of outdoor-to-indoor channel in the $60-\mathrm{GHz}$ band[J]. Journal of communications and information networks, 2016, 1(1): 93-101

\section{Introduction}

The remarkable success of wireless technologies ${ }^{[1]}$ has led to a large demand for mobile communication. To satisfy the increasing demand for data, $5 \mathrm{G}$ (the next generation technology) may support radically different techniques over the current generations. Recent trends in RF technology have enabled the production of CMOS chip of $60-\mathrm{GHz}$ RF technology. The use of higher frequencies and big bands for communication, enable the $60-\mathrm{GHz}$ band (almost 7 $\mathrm{GHz}$ of the spectrum) to be an important part of next- generation technology. The $60-\mathrm{GHz}$ band is used world-wide for ultra-high-capacity point-to-point communications $^{[2]}$. For example, the IEEE802.11ad standard has been defined for $60-\mathrm{GHz}$ band, and products based on this standard are currently available. Thus, the propagation characteristics of the $60-\mathrm{GHz}$ band should be studied in detail. With the advent of $5 \mathrm{G}$ mobile communications ${ }^{[3]}$, a new mode of transmission has appeared: that is O2I (OutdoorTo-Indoor) point-to-point communications.

A recent $5 \mathrm{G}$ white paper presented a brief overview of the channel properties of some traditional channels 
such as $\mathrm{O} 2 \mathrm{O}$ (Outdoor-To-Outdoor), InH (Indoor Hotspot Model). However, the paper states that the outdoor to indoor channel can use the same channel modeling approach and parameters as the $\mathrm{O} 2 \mathrm{O}$ for $\mathrm{UMi}$ (Urban Micro) and Uma (Urban Macro) respectively. This would make the additional building penetration loss the only difference between $\mathrm{O} 2 \mathrm{O}$ and $\mathrm{O} 2 \mathrm{I}$.

Conducting a channel measurement campaign is an extremely important method to better understand the characteristics of a $\mathrm{mm}$-Wave channel. Many research teams and universities have measured other $\mathrm{mmW}$ channel. In this paper, we conduct some special experiments to study the characteristics of $60-\mathrm{GHz}$ outdoor-to-indoor propagation. We analysis the channel statistics results and draw a conclusion.

Our main contributions are as follows.

1) We take some measurements for various materials ${ }^{[4]}$. From the result of measurement, we find some special phenomenon. By analyzing the results, we explain the phenomenon of uneven penetration measurement.

2) In addition, the small-scale character ${ }^{[5]}$ of the two conditions for whether a door is open or closed is also studied. We compare the two measurements for different materials in different scenarios. By analyzing the characteristics of the mutipath of $60-\mathrm{GHz}$, we conclude that O2I channel does not add new multipaths.

3) In the final section of this paper, Our result show that an $\mathrm{O} 2 \mathrm{I}$ channel should be just an $\mathrm{O} 2 \mathrm{O}$ channel or InH channel, and that they add a penetration loss of material.

\section{Measurement system}

In this section, we will explain the two measurement systems, the measurement setup, and its basic principle including the parameters that influence the channel measurement. Before the measuring, a calibration is necessary for our study. Different methods for our measurement will be presented.

\subsection{Measurement setup}

The setup to measure the penetration loss is depicted in Fig.1. The measurement system utilizes an analog signal generator on transmitter and a spectrum analyzer on the receiver. The system is able to measurement a frequency range of 57 to $64 \mathrm{GHz}$.

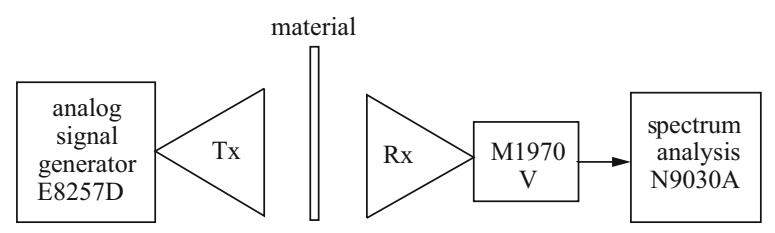

Figure 1 Schematic diagram of the penetration loss measurement

During the measurement, a sweep frequency is created by signal generator E8257D, which cover the measurement range. The output power of the RF signal is $0 \mathrm{dBm}$. In the receiver the RF signal is downconverted using a waveguide harmonic mixers M1970v lead to IF signal. The IF signal is measured by a spectrum analysis.

The measurement setup for a small scale is depicted in Fig.2. We use two mixers to down-convert the signal, and two horn antennas with a gain of 25 $\mathrm{dBi}$ and its HPBW is $30^{\circ}$. The measured data is stored and processed using MATLAB ${ }^{[6]}$.

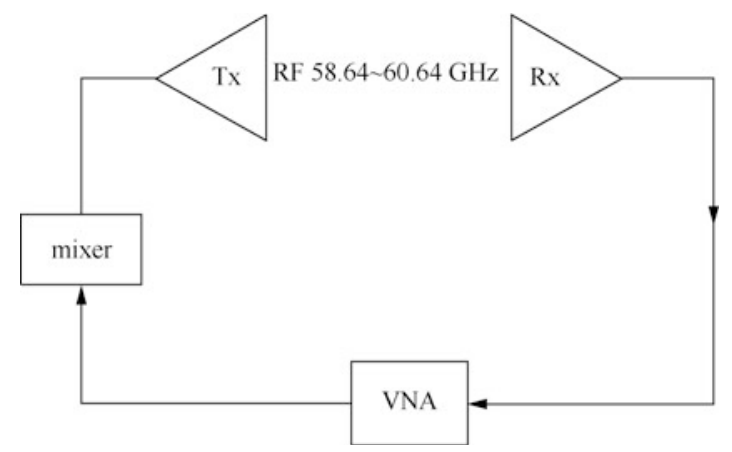

Figure 2 Schematic diagram of small scale measurement 


\subsection{Measurement principle}

The measurement principle of penetration loss satisfies a far-field antenna. The principle can be describe using the formula $d \geqslant 2 D^{2} / \lambda$. For $60-\mathrm{GHz}$, the wavelength is $5 \mathrm{~mm}$, In the formula, $D$ is the max antenna size, which for our antenna is $10 \mathrm{~cm}$. Thus, we can get that, the distance- $d$ meet the principle ${ }^{[7]}$ $d \geqslant 2 \times 0.05^{2} / 0.005=1$ is necessary.

The measurements were taken in different place to ensure the accuracy of results. We put some absorbing material around the measuring instruments to absorb the reflection path. During the measurement, the antenna was statistic.

By measure two conditions (open door and closed door), we can get two sets of data, $P L_{\mathrm{O}}$ for the data of the open door, and $P L_{\mathrm{I}}$ for the close door. so the final penetration loss as follows:

$$
P L=P L_{\mathrm{O}}-P L_{\mathrm{I}} .
$$

The measurement principle of the smale scale test is to simulate a wideband signal through a channel under test for a certain period of time, and to measure the amplitude and phase of the VNA (Vector Network Analyzer) output. Since the VNA can measure ${ }^{[8]}$ the complex amplitude at any frequency, we can determine the $S_{12}$ parameter, which is the same as sampling the channel transfer function $H(f)$. After the VNA finishes a frequency sweep, $N_{\text {points }}$ complex amplitudes and phases of the frequencies $f=i \cdot f_{\text {step }}+f_{\text {star }}\left(i=0-N_{\text {points }}-1\right)$ are available. The $f_{\text {start }}, f_{\text {step }}$, and $N_{\text {points }}$ represent the starting measured frequency, the sweep frequency step, and the number of measured frequencies.

For a better understanding of the properties of the measured impulse, we use an aperture function of the VNA from the aspect of communication theory. The channel transfer function measured by the VNA can be given by the formula as follows:

$$
H_{\mathrm{VNA}}=H_{\mathrm{W}}(f) \cdot B \text {. }
$$

$$
\operatorname{rect}\left(\frac{f}{B}\right) \sum_{i=-\infty}^{\infty} \delta\left(f-i \cdot f_{\text {step }}\right) \cdot H\left(f_{\mathrm{C}}\right) .
$$

The original channel transfer function is represented by $H\left(f_{\mathrm{C}}\right)$ and $f_{\mathrm{C}}=f_{\text {start }}+B / 2$, and the step of sampled is $f_{\text {step }}$. The IFFT-window $H_{\mathrm{w}}(f)$ was an option in post processing, and the operator's "rect" represents the rectangular function. The time-domain channel impulse response

$$
\begin{aligned}
h_{\mathrm{VNA}}(t)= & h_{\mathrm{W}}(t) \otimes \operatorname{sinc}(\pi B t) \otimes \sum_{i=-\infty}^{\infty} \delta\left(t-\frac{i}{f_{\text {step }}}\right) \otimes(h(t) . \\
& \left.\exp \left(-\mathrm{j} 2 \pi f_{\mathrm{C}} t\right)\right) .
\end{aligned}
$$

The operator " $\otimes$ " represent the convolution, and the final impulse response is influenced by both the IFFT window $h_{\mathrm{w}}(f)$ and the $\operatorname{sinc}(\pi B t)$ term.

By use the IFFT and some suitable methods to proceeding the date of $H_{\mathrm{VNA}}(f)$, the time domain transfer function $h(t)$ will be obtained.

\subsection{Calibration}

Initially, the result for the penetration $\operatorname{loss}^{[9]}$ is also calibrated by Eq.(1). The transfer function $H_{\mathrm{VNA}}(f)$, it consists of the particular function of the measurement chain as follows:

$$
H_{\mathrm{VNA}}=H_{\mathrm{v}}(f) \cdot H_{\mathrm{M}}(f) \cdot H_{\mathrm{C}}(f) .
$$

$H_{\mathrm{v}}(f)$ and $H_{\mathrm{M}}(f)$ represent the transfer function of the VNA front ends and mixer. $H_{\mathrm{C}}(f)$ represent all transfer functions of all cables used, and $H_{\mathrm{C}}(f)$ represents the actual transfer function of the actual channel. Since $H_{\mathrm{C}}(f)$ is the only function of interest, a calibration step is necessary to eliminating the other functions.

A back-to-back calibration can be done after the measurement. In the calibration measurement, Tx and $\mathrm{Rx}$ can be connected to each other via a waveguide. The calibration data $H_{\mathrm{CAL}}(f)$ can be obtained from the calibration and consists of two terms $H_{\mathrm{V}}(f) \cdot H_{\mathrm{M}}(f)$. $H_{\mathrm{C}}(f)$ and the fixed attenuation value $(30 \mathrm{~dB})$. Thus, the final calibrated channel transfer is 


$$
H_{\mathrm{TF}}(f)=H_{\mathrm{VNA}} / H_{\mathrm{CAL}}(f)+30 .
$$

In order to obtain omnidirectional channel information, the receiver must collect data for a $360^{\circ}$ rotation with a sampling scale of $5^{\circ}$. By fitting the different perspectives of information, we can obtain substantial omnidirectional channel information.

\subsection{System analysis}

Before we start our measurement, it is necessary to analyze the validity and accuracy of our channel measurement system.

The calibration of the system and some post processing of system (which were described in the preamble), that two term can make sure the data we measured is validity. Except the methods of processing data, we sample data for $360^{\circ}$ with an angular step of $5^{\circ}$ for each receiver site.

The accuracy of our measurement system requires that we develop an automatic control system for angle rotation.

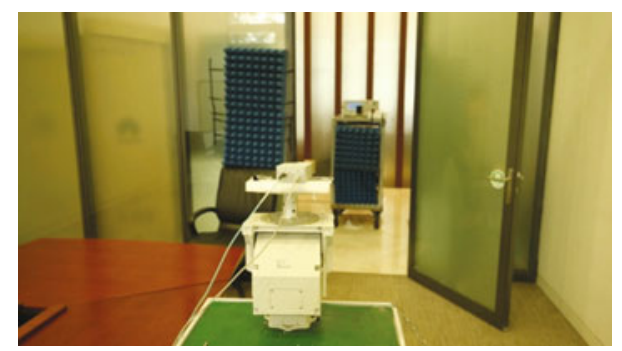

Figure $3 \mathrm{Rx}$ and $\mathrm{Tx}$ locations for the penetration loss measurement

\section{Measurement scenarios}

Two channel measurements were performed. One was for the penetration and the other was for a small scale.

\subsection{Penetration loss measurement}

These measurements were conducted in Huawei 2012
Lab, which is located in Chengdu, Sichuan. In these measurements, Tx and Rx were $1.5 \mathrm{~m}$ high, and their positions can be seen in Fig.3. The distance between Tx and $R x$ is $3 \mathrm{~m}$.

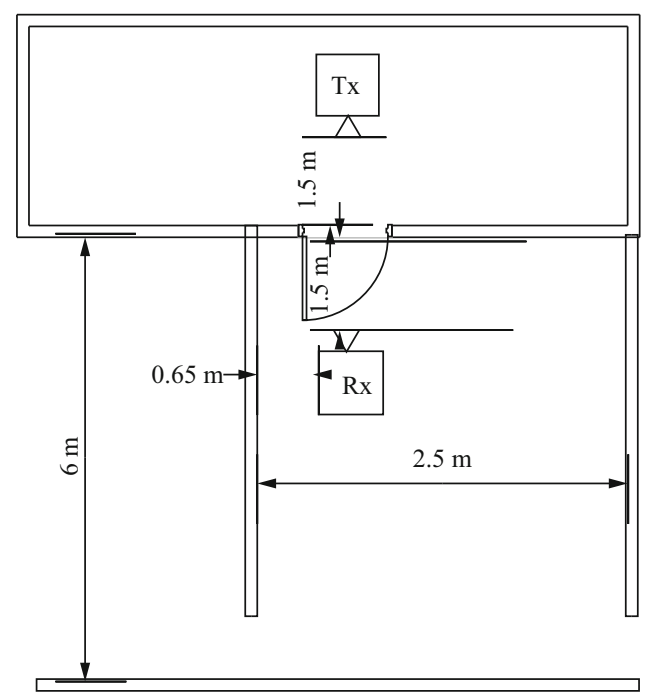

Figure 4 Schematic diagram of measurement of meeting room with wooden doors

\subsection{Small scale measurement}

These measurements were conducted in two different locations: a meeting room with doors (see the schematic in Fig.4), and a meeting room with glass (see the schematic in Fig.5). For these measurements, Tx and Rx were $1.5 \mathrm{~m}$ high. The distance between Tx and $\mathrm{Rx}$ was $3 \mathrm{~m}$.

\section{Measurement results}

The calibrated data of the penetration loss are presented in Fig.6. We measured the penetration loss of three common materials: wooden doors, monolayer-glass and double-height transparent glass.

In observing the test results found in the $50 \sim 67 \mathrm{GHz}$ band, the penetration loss for different materials exhibited different degrees of volatility, and with 
increasing frequency have a rising trend. Given this wide band with increasing penetration loss and volatility, we can explain this phenomenon using electromagnetic shielding theory.

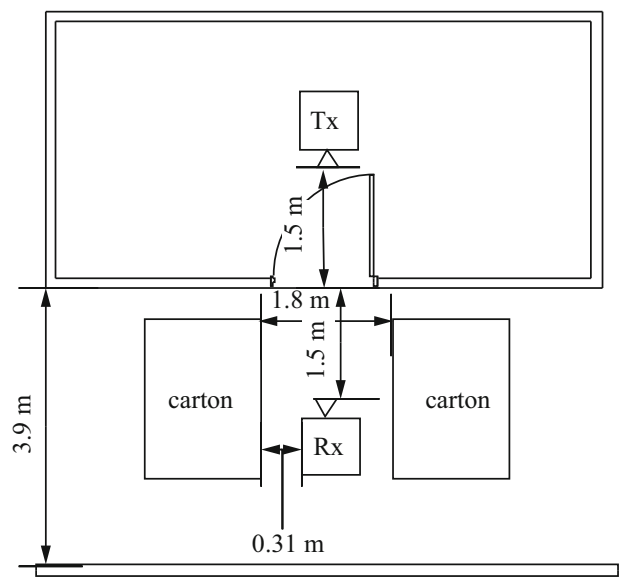

Figure 5 Schematic diagram of measurement of meeting room with glass doors

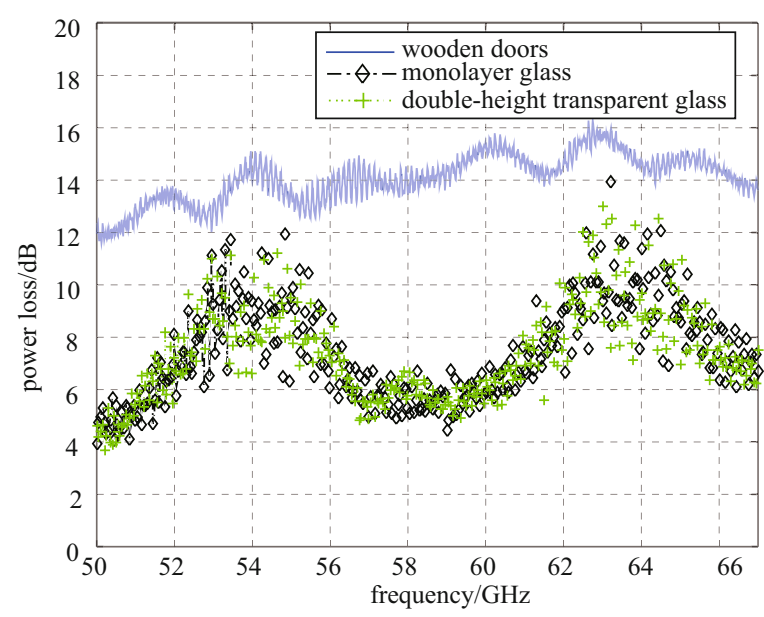

Figure 6 Penetration of different materials

In the electromagnetic field, we can interpret this phenomenon as a shielding effect of a square object. An electromagnetic shielding effect can be divided into two parts: an absorption shield and a reflective shield, its formula is as follows:

$$
S=S_{n} S_{0} .
$$

Absorption shielding is a result of the heatshielding effect of an electromagnetic shielding eddy. It increases with the frequency and shield thickness. The reflective shield is determined by the metal shield and shield potter vitro medium degree of inconsistency. The greater the difference in the waveguide properties of the two materials, the stronger the effect of the reflection shielding. Finally, the higher the frequency, the greater the fluctuation in reflective shield. In the Fig.7, we can see this clearly.
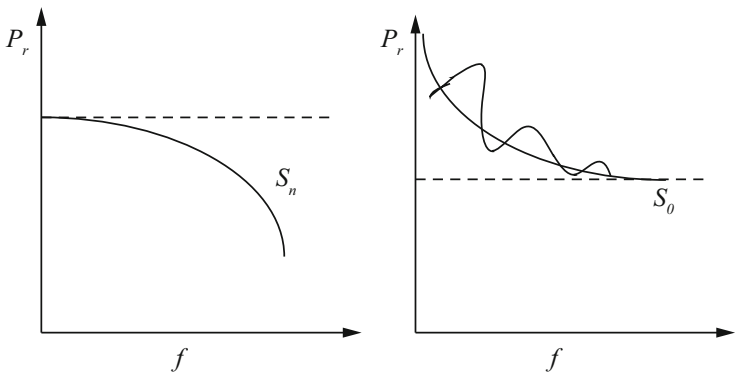

Figure 7 Frequency variation for absorption and reflection shield

The difference between the $\mathrm{O} 2 \mathrm{O}$ channel or the $\mathrm{InH}$ channel and the O2I channel is that the signal crosses some material. Thus, a test for two situations (open or closed door). We tested two scenarios, (Fig.4 and Fig.5). Finally, the processed data for two materials are presented in Fig.8 to Fig. 13.

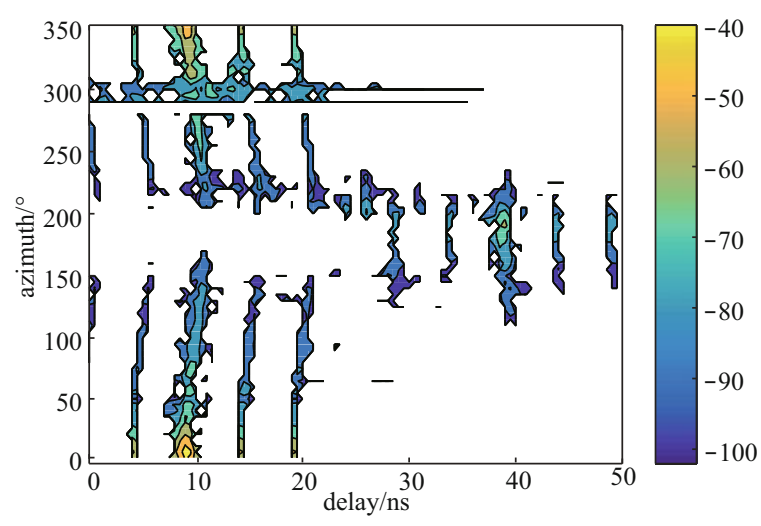

Figure 8 Delay profile for opening the wooden door

The distance between the Tx and Rx horn antennas is $3 \mathrm{~m}$, so the first path must be within $9 \mathrm{~ns}$ in LOS channel. The IFFT transport, may introduce interference before the first path. Thus, we only 
analyze the path after the first path. By comparing the two situations (opened and closed) in Fig.8 and Fig. 9, the main three path have the same delay while only have different power.

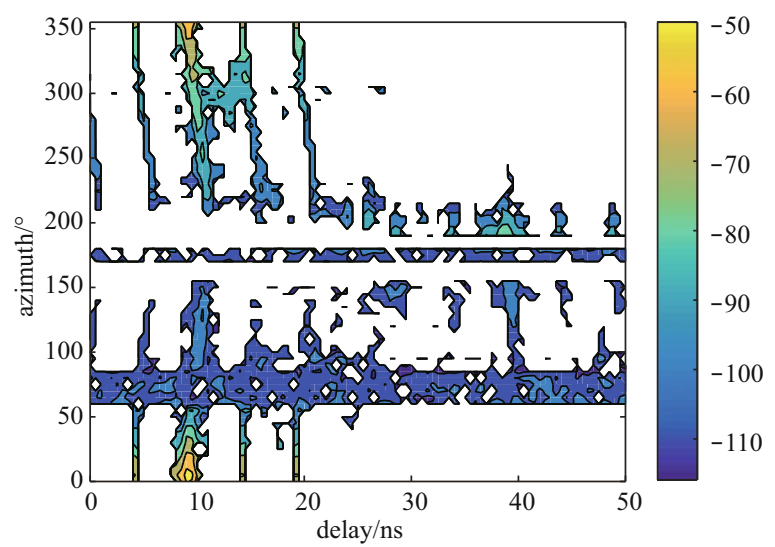

Figure 9 Delay profile of for closing the wooden door

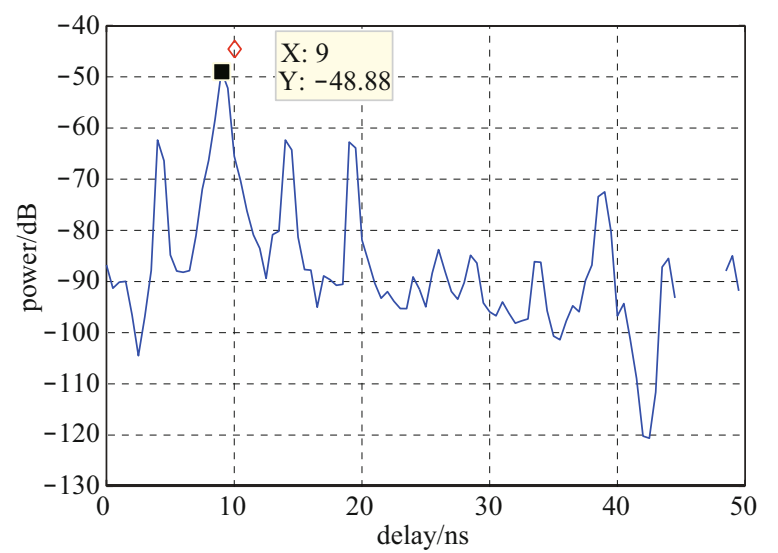

Figure 10 PDP of omnidirectional channel in situation with open wooden door

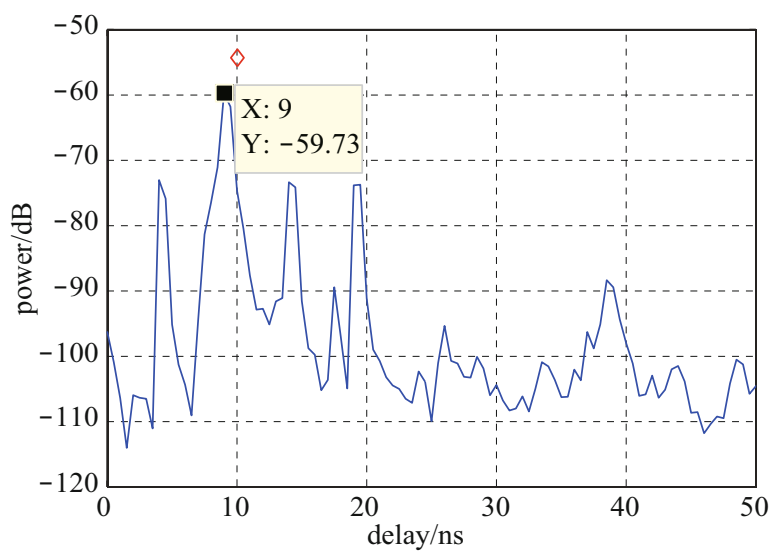

Figure 11 PDP of omnidirectional channel in close wooden door situation
The PDP (Power Delay Profile) for each situation is detailed in Fig.10 and Fig.11. By extracting three major diameters of power and delay, we obtain the information in Tab.1 and Tab.2. The difference between main the main three paths of the two situations is just the power. The penetration loss of the wooden door is $12 \sim 16 \mathrm{~dB}$ in the wideband of 50 67 GHz. The power of the main path in the two situations, differ by $11 \mathrm{~dB}$, which is similar to the penetration loss of the wooden door.

Tab.3 lists the delay spread and angle spread of the two situations. When the wooden door is closed, the spread shrinks somewhat.

Table 1 Main three path profile of open wooden door

\begin{tabular}{cccc}
\hline path number & first path & second path & third path \\
\hline delay/ns & 9 & 14.5 & 19.5 \\
power/dB & -48.88 & -62.35 & -62.74 \\
\hline
\end{tabular}

To further validate our measurement results, a similar measurement was conducted for the monolayer-glass door (see Fig.5), and the results are shown in Fig.12 and Fig.13. The delay profile is similar to that for the wooden door, and all profiles have three main path.

Table 2 Main three-path profile of closed wooden door

\begin{tabular}{cccc}
\hline path number & first path & second path & third path \\
\hline delay/ns & 9 & 14.5 & 19.5 \\
power/dB & -59.73 & -73.35 & -73.82 \\
\hline
\end{tabular}

Table 3 Spread parameter of wooden door

\begin{tabular}{ccc}
\hline spread parameter & open & close \\
\hline delay spread/ns & 9.2 & 6.49 \\
angle spread/ & 18.1141 & 14.8615
\end{tabular}

For a detailed data describe of PDP, similar results can be seen in Fig.14 and Fig.15. It is very clear that the three main paths are similar to those of the wooden door situation. 


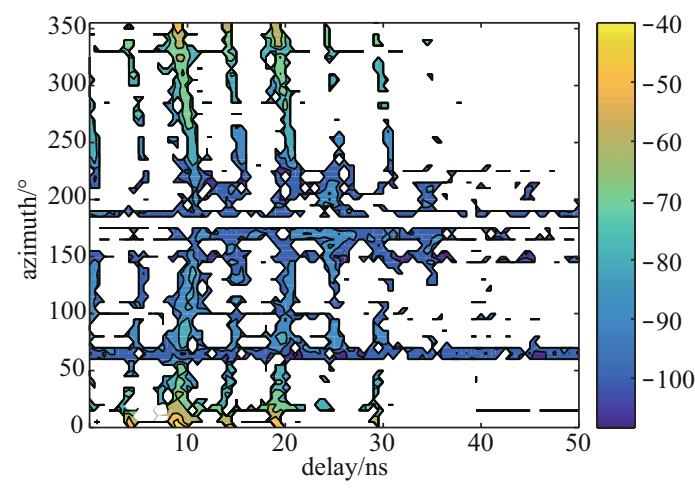

Figure 12 Delay profile of open monolayer-glass door

Tab.4 lists the delay spread and angle spread for the two situations for the monolayer-glass door. The different between the two material is that the spread of monolayer-glass would be little small. By comparing the tests of the two materials of in different scenarios, we conclude that our measurements have a certain credibility.

In the measurement result for the two scenarios, the different scenario provide different channel environments but the results are similar. we conclude that, the new propagation channel (outdoor-to-indoor) does not generate new path

Compared with a traditional channe ${ }^{[10]}$, and just adds some penetration loss. Some small changes can be considered, such as having a smaller spread in the delay and angle $\mathrm{e}^{[11]}$.

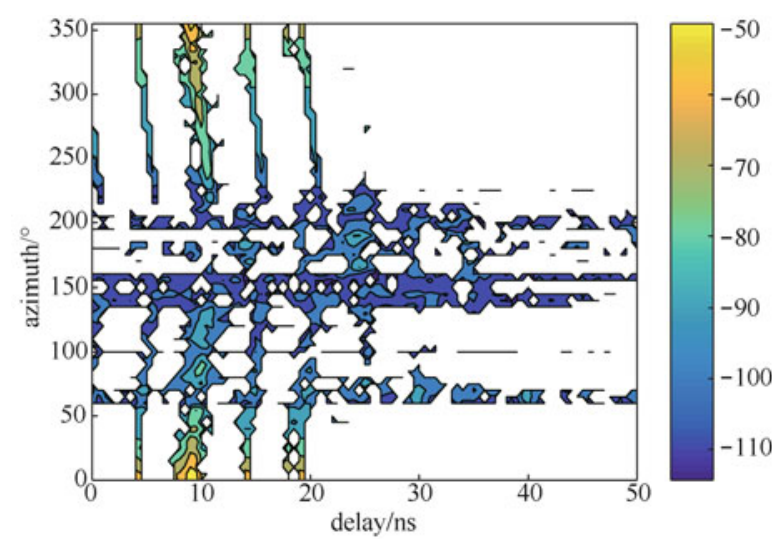

Figure 13 Delay profile of close monolayer-glass door

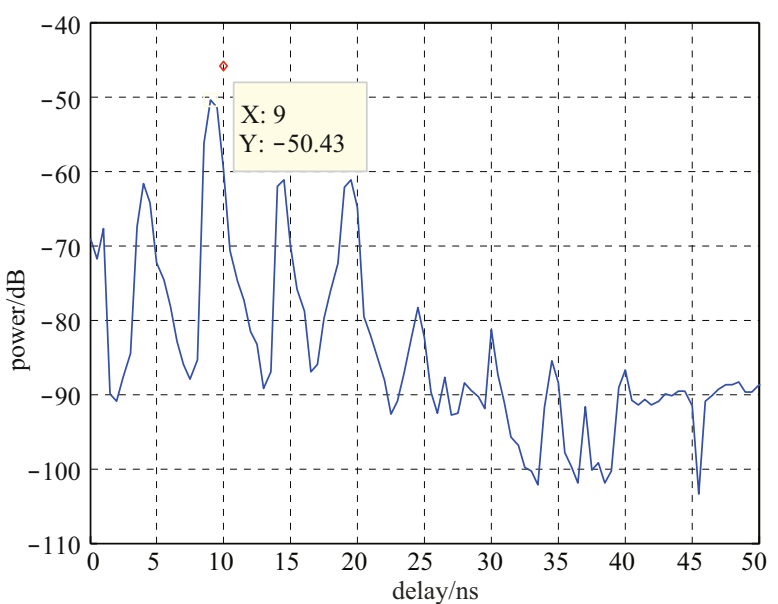

Figure 14 PDP of the omnidirectional channel in open Monolayer-glass door situation

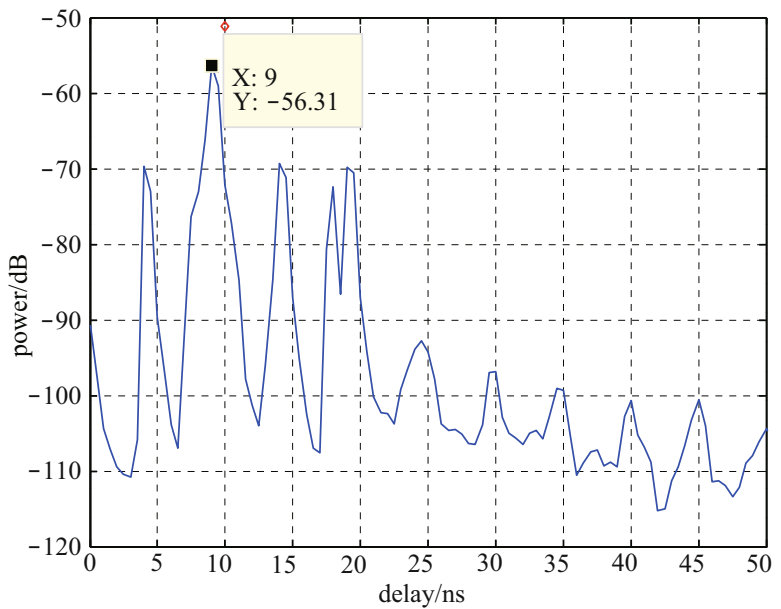

Figure 15 PDP of the omnidirectional channel in open Monolayer-glass door situation

Table 4 The spread parameter of monolayer-glass door two situations

\begin{tabular}{ccc}
\hline spread parameter & open & close \\
\hline delay spread/ns & 4.23 & 2.79 \\
angle spread/ & 16.2575 & 13.4395 \\
\hline
\end{tabular}

\section{Conclusion}

In this paper, two measurement systems for penetration loss and small-scale channel measurement 
were presented. The measurement system for penetration utilizes a signal source as a transmitter and a spectrum analysis as the receiver. The measurement system of small scale uses a VNA as a transmitter and receiver. For the two measurement system, we use different principles to calibrate and measure data.

We determined the penetration loss of three materials and explained the phenomenon of fluctuation with increasing frequency. Finally, a test of small scales of different materials in different scenarios was conducted. The tests prove that the O2I channel does not generate new paths and only adds some penetration loss.

\section{References}

[1] AKDENIZ M R, LIU Y, SAMIMI M K, et al. Millimeter wave channel modeling and cellular capacity evaluation[J]. IEEE journal on selected areas in communications, 2014, 32(6): 11641179 .

[2] MORAitis N, CONSTANTINOU P. Measurements and characterization of wideband indoor radio channel at $60 \mathrm{GHz}[\mathrm{J}]$. IEEE Transactions on Wireless Communications, 2006, 5(4): 880-889.
[3] RAPPAPORT T S, SUN S, MAYZUS R, et al. Millimeter wave mobile communications for $5 \mathrm{G}$ cellular: it will work![J]. IEEE access, 2013, 1(1): 335-349.

[4] ANDERSON C R, RAPPAPORT T S. In-building wideband partition loss measurements at 2.5 and $60 \mathrm{GHz}[\mathrm{J}]$. IEEE transactions on wireless communications, 2004, 3(3): 922-928.

[5] BEN-DOR E, RAPPAPORT T S, QIAO Y, et al. Millimeter-wave $60 \mathrm{GHz}$ outdoor and vehicle AOA propagation measurements using a broadband channel sounder[C]//IEEE Global Telecommunications Conference, 2013, 263(4): 1-6.

[6] RAPPAPORT T S, BEN-DOR E, MURDOCK J N, et al. $38 \mathrm{GHz}$ and $60 \mathrm{GHz}$ angle-dependent propagation for cellular \& peer-topeer wireless communications[C]//EEE International Conference on Communications (ICC), Ottawa, Canada, 2012: 4568-4573.

[7] SMULDERS P, WAGEMANS A G. Wideband indoor radio propagation measurements at $58 \mathrm{GHz}[\mathrm{J}]$. Electronics letters, 1992, 28(13): 1270-1272.

[8] MANABE T, SATO K, IHARA T. Measurement of complex refractive index of soda-lime glass at $60 \mathrm{GHz}$ by vector-networkanalyser-based scatterometer[J]. Electronics letters, 1992, 28(14): 1354-1355.

[9] CORREIA L M, FRANÇÊS P O. Estimation of materials characteristics from power measurements at $60 \mathrm{GHz}[\mathrm{C}] / / \mathrm{IEEE}$ International Symposium on Personal, Hague, Netherlands 1994: 510-513.

[10] MACCARTNEY G R, ZHANG J, NIE S, et al. Path loss models for $5 \mathrm{G}$ millimeter wave propagation channels in urban microcells[C]//Globecom IEEE Global Communications Conference, Atlanta, USA, 2013:

[11] TSE D, VISWANATH P. Fundamentals of wireless communication[M]. Cambridge university press, 2005.

\section{About the authors}

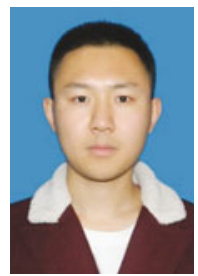

CHENG Long [corresponding author] was born in Kaifeng. He received the B.E. in communication engineering from. the PLA Information Engineering University in 2007. He is now working toward the M.S. degree in electrical engineering at the University of Electronic Science and Technology of China. His current research interests focus on millimeter-wave communications. (Email: chenglong_office@163.com)

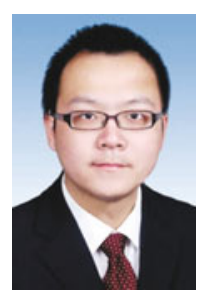

BAO Wencheng was born in Nei Jiang . He received the B.E. degree in communications engineering from Shanghai university. He is studying for a master's degree in electrical engineering at the University of Electronic Science and Technology of China. His current research interests focus on millimeter-wave communications. (Email: 18215549550@139.com)

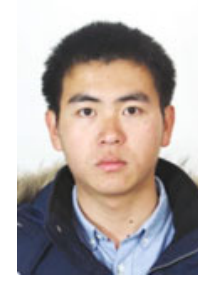

LIU Ning was born in Shangqiu. He received the B.S. degree in comunication engineering from University of Electronic Science and Technology of China in 2015. He is currently working toward a M.S. degree in electrical engineering at the University of Electronic Science and Technology of China. His research interests include wireless communication, $60 \mathrm{GHz}$ millimeter wave propagation and channel modeling. (Email: 201521260150@std.uestc.edu.cn )

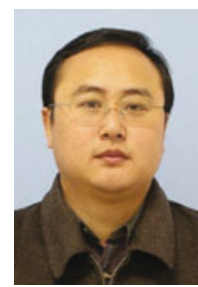

YUE Guangrong received the Ph.D. degrees in communication and information system from University of Electronic Science and Technology of China, Chengdu, China, in 2006. He was a Postdoctoral Fellow at the Department of Electrical Engineering and Computer Science at the University of California Berkeley, from 2007-2008. He has been a faculty member in Telecommunications Engineering at the 
University of Electronic Science and Technology of China since 2008. Dr. Yue is a member of IEEE. His major research interests include wireless communication theory, short range communications, and adaptive signal processing. (Email: yuegr@uestc.ed.cn)

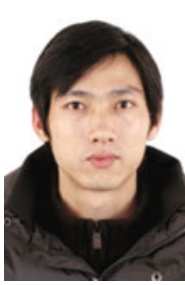

ZOU Xianbing was born in JiangXi province of China, in 1974. He received the M.S. degree in electromagnetic field and microwave technology from University of Electronic Science and Technology of China (UESTC), China. He is now an associate researcher at UESTC. His research interests include RF technique for wideband wireless communication systems and antenna technique for self-adaptive waveform array. (Email: zouxb@uestc.edu.cn)

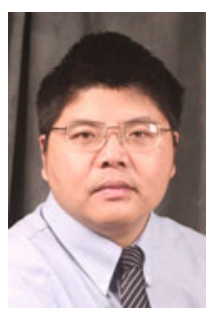

QIU Robert Caiming has nearly 20 years of teaching and research in academia, industry and startup with diverse research experience in wireless communications and networks, wireless (and remote) sensing, Big Data and Smart Grid. His professional experiences outside the academia include GTE Labs (now Verizon Wireless), Bell Labs (Lucent Technologies) and the startup. He served as founder, CEO and President for Wiscom Technologies Inc. that grew to a total of $30+$ staff and whose assets were sold to Intel. He is a US citizen (since 2001).
He joined Tennessee Technological University (TTU) in 2003. In 2008, he was tenured and promoted to a full professor at TTU. He was also the principal investigator for a Congressional Earmark Project that has the planned budget of $\$ 5$ millions in three phases, although only the first phase was actually funded due to the change of policy in US Congress. He served as the founding coordinator for two college-level focus areas: (1) Smart Grid and (2) Big Data. Dr. Qiu has attracted a total funding over $\$ 3$ millions from diverse sources, including NSF, ARO, ONR, AFOSR and AFRL. He spent four summers in federal defense laboratories (AFRL and NRL).

Before coming to TTU, he spent three years to found his startup (as CEO and President). At this leadership position, Wiscom Technologies Inc. raised a total of $\$ 8.5$ millions from Wall Street Investors and AMD (series A, series B). Wiscom designs and markets chipsets for 3G WCDMA cell phones. Prior to his startup experience, he spent nearly 5 years in industrial labs (GTE Labs and Bell Labs), to conduct applied research in wireless cellular industry. In GTE Labs, he participated in the first CDMA field trial jointly conducted by AT \& T, GTE, and Qualcomm. CDMA technology is the industrial standard for the second-generation $(2 \mathrm{G})$ and third-generation $(3 \mathrm{G})$ wireless system. At Bell Labs, he was one of the pioneers in designing the core physical layer system algorithms for the 3G WCDMA base transceiver station that turns into multi-billion dollars business later for Lucent. 